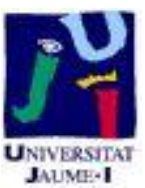

Título artículo / Títol article: Positivity of discrete singular systems and their stability: An LP-based approach

Autores / Autors

M. Ait Rami a, D. Napp

Revista:

Automatica

Versión / Versió:

Preprint del autor

Cita bibliográfica / Cita

RAMI, Mustapha Ait; NAPP, Diego. Positivity of discrete bibliogràfica (ISO 690): singular systems and their stability: An LP-based approach. Automatica, 2014, vol. 50, no 1, p. 84-91.

url Repositori UJI:

http://hdl.handle.net/10234/120187 


\title{
Positivity of discrete singular systems and their stability: An LP-based approach
}

\author{
M. Ait Rami ${ }^{\text {a }}$, D. Napp ${ }^{\mathrm{b}}$ \\ ${ }^{a}$ Departamento de Ingenieria de Sistemas y Automatica, Univ. of Valladolid, 47005 Valladolid, Spain. \\ ${ }^{\mathrm{b}}$ Department of Mathematics, University Jaume I, Castellón 12071, Castellón, Spain.
}

\begin{abstract}
In this paper we present an efficient approach to the analysis of discrete positive singular systems. One of our main objectives is to investigate the problem of characterizing positivity of such systems. Previously, this issue was not completely addressed. We provide easily checkable necessary and sufficient conditions for such problem to be solved. On the other hand, we study the stability of discrete positive singular systems. Note that this is not a trivial problem since the set of admissible initial conditions is not the whole space but it is represented by a special cone. All the conditions we provide are necessary and sufficient, and are based on a reliable computational approach via linear programming.
\end{abstract}

Key words: Singular systems; positive systems; input-ouput positivity; stability; linear programming

\section{Introduction}

Over the past few years, singular systems (also referred to as descriptor systems, semi-state systems, implicit systems, differential-algebraic systems, or generalized state-space systems) have constantly gained a great interest. This kind of systems naturally appears in many practical areas such as robotics, compartmental systems, circuit systems, Leontief dynamic models, etc..., see $[20,22,30,32]$. Their solutions and fundamental properties such as stability and controllability have been fully studied. Important developments took place in the 80 's, see for instance the survey paper $[24]$ and $[7,12]$. In the last three decades, some interesting monographs entirely devoted to many topics of this type of systems have been presetnted $[7,12,14,22,30,23,35]$ along with a vast amount of contributions extending the framework of standard systems to deal with stabilization and robustness. These intensive developments are a testimony of the vitality and the maturity of this field that remains an area of active research, see for instance recent works among others $[3,16,34,31]$.

In this paper, our focus is on discrete singular systems under positivity constraint on their states. This is inherent to many real-world systems for which the states are

Email addresses: musta.ait.rami@gmail.com (M. Ait Rami), diegonapp@gmail.com (D. Napp). intrinsically nonnegative since they can represent real physical quantities such as concentrations, level and volume of matter transfer, size of populations, etc. Singular systems which have nonnegative states whenever the initial conditions are nonnegative are referred to as positive systems $[15,21,26]$ or evenly as nonnegative systems [11].

Although many fundamental issues have been wellinvestigated for standard singular systems and, in particular for standard positive systems, they have not been sufficiently investigated for the specific class of positive singular systems. To the best of our knowledge few works on such systems can be found in the literature $[4,5,10,18,19,29,36]$. These works are based on a common standing, but unnecessary, assumption for positivity of a singular system. That is, the matrix that represents the projector on the set of admissible initial conditions, is nonnegative. Most of the reported results have focused on other fundamental properties such as reachability and controllability. The stability issue was considered only in $[36,1]$. In this paper, unlike the previous reported results on discrete positive singular systems, positivity is fully investigated without any unnecessary assumption. Note that, in general, numerically checking positive invariance for a particular set of initial conditions even for a standard linear system can be quite complicated. This fact also applies to the stability analysis for a given set of initial conditions. 
This issue has been investigated in [33] and [28] for LTI systems with respect to a closed convex pointed cone for which necessary and sufficient conditions for positive invariance and stability have been provided. However, the proposed results are rather theoretical and cannot be checked numerically. In the case of positive singular systems we have to deal with a specific conic set of the form $\operatorname{im}(P) \cap \mathbb{R}_{+}^{n}$, where the matrix $P$ represents the projector on the admissible set of initial conditions. For such set the stability issue is also addressed. The proposed approach is numerically appealing for checking positivity and stability of a given discrete singular system. All the proposed conditions are necessary and sufficient and can be checked by using Linear Programming (LP).

The structure of the paper is as follows. Section 2 gives the necessary background on singular systems. Section 3 is concerned with the positivity of discrete singular systems for which some characterizations are provided together with an illustrative example. Section 4 deals with the stability issue. In Section 5 the notion of internal positivity is investigated. Section 6 gives some conclusions.

Notation: $\mathbb{R}_{+}^{n}$ denotes the nonnegative orthant of the $n$ dimensional real space $\mathbb{R}^{n}$ and $\dot{\mathbb{R}}_{+}^{n}$ its interior. A real matrix (or a vector) $M=[M(i, j)]$ is called nonnegative, denoted by $M \geq 0$, if all its components are nonnegative (i.e., $M(i, j) \geq 0$ ); analogously, a positive matrix or vector is denoted by $M>0$ if its components are strictly positive. $M^{+}$is used to denote the Moore-Penrose pseudoinverse of the matrix $M$ and $\sigma(M)$ its spectrum.

\section{Solvability}

This section provides preliminary results regarding the existence and characterization of the solution of the following time-invariant homogeneous singular system

$$
\operatorname{Ex}(k+1)=A x(k)
$$

where $E, A \in \mathbb{R}^{n \times n}$. In contrast to standard linear systems for which $E$ is invertible, system (1) may not possess a solution for arbitrary initial conditions.

Definition 2.1 The set of initial conditions for which system (1) has a solution is called the set of admissible initial conditions.

The characterization of the admissible set of initial conditions with their associated trajectories involves the Drazin inverse. Hence, we first present some basic properties of this kind of inverse (see $[8,13]$ for more details). For any matrix $M \in \mathbb{R}^{n \times n}$ there always exists a unique matrix $M^{D}$, which is called the Drazin inverse of $M$, such that $M^{D} M=M M^{D}, M^{D} M M^{D}=M^{D}$ and $M^{D} M^{\nu+1}=M^{\nu}$, where $\nu$ is the smallest nonnegative integer such that $\operatorname{rank}\left(M^{\nu}\right)=\operatorname{rank}\left(M^{\nu+1}\right)$. In [22], it is shown how the Drazin inverse can be computed, see also $[9,37,38]$ for more details on this issue. One way to compute it is the following: by using the Jordan canonical form, any matrix $M$ can be decomposed as

$$
M=T\left(\begin{array}{ll}
C & 0 \\
0 & N
\end{array}\right) T^{-1},
$$

where $C$ is invertible and $N$ is a nilpotent matrix. Then, its Drazin inverse is given by

$$
M^{D}=T\left(\begin{array}{cc}
C^{-1} & 0 \\
0 & 0
\end{array}\right) T^{-1} .
$$

The following result presents a characterization for the solvability of system (1). In [7], a precise explicit solution to system (1) has been given (see also [22]).

Theorem 2.2 [7] The singular system (1) admits a unique solution for each admissible initial condition if and only if $(E, A)$ is regular (i.e., there exists a $\lambda \in \mathbb{C}$ such that $(\lambda E-A)^{-1}$ exists). Moreover, the set of admissible initial conditions is given by $\mathcal{X}_{0}:=i m\left(\widehat{E}^{D} \widehat{E}\right)$ and the solutions of (1) have the following form

$$
x(k)=\left(\widehat{E}^{D} \widehat{A}\right)^{k} \widehat{E}^{D} \widehat{E} v,
$$

where $v$ is an arbitrary vector in $\mathbb{R}^{n}$, the matrices $\widehat{A}$ and $\widehat{E}$ are given by,

$$
\widehat{E}=(\lambda E-A)^{-1} E, \widehat{A}=(\lambda E-A)^{-1} A,
$$

with $\lambda$ any complex number such that $(\lambda E-A)^{-1}$ exists, and $\widehat{E}^{D}$ is the Drazin inverse of $\widehat{E}$.

Remark 2.3 Theorem 2.2 summarizes the results of Theorem 3.6.1 and Theorem 3.6.2 in [7]. Based on this theorem, one can see that the trajectory (4) is the solution to the difference equation $x(k+1)=\widehat{E}^{D} \widehat{A} x(k)$ with $x(0)=\widehat{E}^{D} \widehat{E} v \in i m\left(\widehat{E}^{D} \widehat{E}\right)$. Note that the solution (4) does not depend on the value of $\lambda$ used to define $\widehat{E}$ and $\widehat{A}$. For more details see [7,22].

According to Theorem 2.2 we assume throughout the rest of the paper that $(E, A)$ is regular.

In the sequel, we shall make use of some useful properties of the matrices $P:=\widehat{E}^{D} \widehat{E}$ and $\bar{A}=\widehat{E}^{D} \widehat{A}$ that characterize the admissible set of initial conditions. Such properties are presented in the following result.

Lemma 2.4 [1, Lemma 3.2] The following properties hold true. 
(i) $P$ is idempotent or a projector (i.e., $P^{2}=P$ ).

(ii) $P \bar{A}=\bar{A} P=\bar{A}$.

(iii) For any solution $x(k)$ to system (1) we have

$$
P x(k)=x(k) .
$$

\section{Positivity}

This section deals with the characterization of positivity of system (1). Although the positivity analysis is simple when the set of addmissible initial conditions is the positive orthant, the characterization of positivity for an arbitrary set of initial conditions is not, in general, an easy task. The positive invariance for LTI systems with respect to a given cone has been studied in $[28,33]$. However, the reported results are rather theoretical and cannot be checked numerically. In what follows, we shall investigate computationally sound conditions for positivity of system (1) in connection with the conic set of the form $\operatorname{im}(P) \cap \mathbb{R}_{+}^{n}$. Observe that when the matrix $E$ is nonsingular, system (1) reduces to the standard linear system $x(k+1)=E^{-1} A x(k)$. Obviously, in this case system (1) is positive if and only if $E^{-1} A$ is a nonnegative matrix (i.e., $E^{-1} A \geq 0$ ).

Definition 3.1 We say that system (1) is positive if for any nonnegative admissible initial condition $x(0) \in$ $\mathcal{X}_{0}=i m(P) \cap \mathbb{R}_{+}^{n}$ we have that $x(k) \geq 0$ for all $k \geq 0$.

In order to derive our results for the positivity of system (1) we shall make use of the following lemma which has been first established in [27].

Lemma 3.2 Let $M, N$ be matrices with appropriate sizes. The following statements are equivalent:

(1) $M x \geq 0$ implies that $N x \geq 0$,

(2) there exists $H \geq 0$ satisfying the matrix equation

$$
N=H M
$$

Now, consider the system

$$
\begin{aligned}
x(k+1) & =\bar{A} x(k) \\
x(0) & \in \operatorname{im}(P) .
\end{aligned}
$$

Note that the previous result of Theorem 2.2 and Remark 2.3 show that system (6) and system (1) possess the same set of solutions for any initial condition in the image of $P$. Based on such relationship one can see that the positivity of system (1) is equivalent to the positivity of system (6) for the set $\operatorname{im}(P) \cap \mathbb{R}_{+}^{n}$. Keeping in mind this fact, we are now in a position to characterize the positivity of system (1).

Theorem 3.3 The following statements are equivalent.
(1) System (1) (or (6)) is positive for the set of nonnegative admissible initial condition $\mathcal{S}=i m(P) \cap \mathbb{R}_{+}^{n}$.

(2) There exists a matrix $H$ that satisfies the following conditions

$$
\left\{\begin{array}{l}
H \geq 0 \\
\bar{A}=H P
\end{array}\right.
$$

Proof. $(1 \Rightarrow 2)$ : Let $x(0)=P v$. By assumption, since $x(1)=\bar{A} x(0)$, we have that $\bar{A} P v \geq 0$ if $P v \geq 0$. By Lemma 3.2 there exists a matrix $H \geq 0$ such that $\bar{A} P=$ $H P$. Further, $\bar{A} P=\bar{A}$, by statement (ii) of Lemma 2.4, which yields $\bar{A}=H P$.

$(1 \Leftarrow 2)$ : We have that $x(k+1)=\bar{A} x(k)=H P x(k)$. Then by statement (iii) of Lemma 2.4 one can use the property $P x(k)=x(k)$ and therefore the system reduces to $x(k+1)=H x(k)$. Since $H$ is nonnegative then system (1) is positive.

We next provide an example to illustrate Theorem 3.3. Indeed, despite the fact that $P$ and $\bar{A}$ are not nonnegative, the associated system is positive.

Example 3.4 Let system (1) be given by

$E=\left(\begin{array}{cc}-1 & 1 \\ 1 & -1\end{array}\right)=\left(\begin{array}{cc}-1 & 1 \\ 1 & 1\end{array}\right)\left(\begin{array}{cc}-2 & 0 \\ 0 & 0\end{array}\right)\left(\begin{array}{cc}-0.5 & 0.5 \\ 0.5 & 0.5\end{array}\right)$

and $A=\left(\begin{array}{ll}1 & 0 \\ 0 & 1\end{array}\right)$. In order to check the regularity con-

dition one can choose $\lambda=0$, so that $\widehat{A}=-A$ and $\widehat{E}=-E$. Then, by using equation (3) it is easy to see that $4 \widehat{E}^{D}=\widehat{E}=-E$. This leads to

$$
P=0.5\left(\begin{array}{cc}
1 & -1 \\
-1 & 1
\end{array}\right) \text { and } \bar{A}=0.25\left(\begin{array}{cc}
-1 & 1 \\
1 & -1
\end{array}\right)
$$

Since the nonnegative matrix $H=0.5\left(\begin{array}{ll}0 & 1 \\ 1 & 0\end{array}\right)$ satisfies $\bar{A}=H P$, then by Theorem 3.3 we conclude that this system is positive.

The result of Theorem 3.3 characterizes the positivity of system (1) in terms of the existence of a matrix $H$ that is nonnegative and satisfies a linear equality which can be effectively checked via Linear Programming. However, this characterization can be improved by getting rid of the equality constraint in condition (7) and then obtaining a single linear inequality. To this end, we need the following well-known lemma which is due to Penrose. 
Lemma 3.5 [8] The matrix system $X M=N$ has a solution in the variable $X$ if and only if

$$
N\left(I-M^{+} M\right)=0 .
$$

Moreover, all the solution are given by

$$
X=N M^{+}+D\left(I-M M^{+}\right)
$$

where $D$ is an arbitrary matrix.

We now state the following simplified positivity result.

Theorem 3.6 The following statements are equivalent.

(1) System (1) (or (6)) is positive for the set of nonnegative admissible initial condition $\mathcal{S}=i m(P) \cap \mathbb{R}_{+}^{n}$.

(2) There exists a matrix D such that

$$
\bar{A}+D(I-P) \geq 0
$$

Proof. Obviously, by Theorem 3.3 it is enough to show the equivalence between statement (2) of Theorem 3.3 and statement (2) of Theorem 3.6.

Assume that statement (2) of Theorem 3.3 holds true. That is, the equation $\bar{A}=H P$ is solvable and has a solution $H \geq 0$. First note that the MoorePenrose pseudoinverse of any idempotent matrix $P=P^{2}$ equals $P$, i.e., $P^{+}=P$. By applying Lemma 3.5 to the equation $\bar{A}=H P$ we have that $H=\bar{A} P+D\left(I-P P^{+}\right)$, for some matrix $D$. Since $\bar{A} P=\bar{A}$ and $P P^{+}=P^{2}=P$, we readily obtain statement (2) of Theorem 3.6.

For the converse implication denote $H:=\bar{A}+D(I-P)$. Then, by using the statements (i) and (ii) of Lemma 2.4 it is straightforward to verify that $\bar{A}=H P \geq 0$.

Next, we show how the derived positivity conditions can be reexpressed in terms of standard Linear Programming. For this purpose, it suffices to use the Kronecker product $\otimes$ and the vec operation (which consists of taking the columns of a given matrix from left to right and stack them one above the other). Also, for matrices $M, N$ and $X$ with appropriate sizes, one can make use of the following well-known identity

$$
\operatorname{vec}(M X N)=\left[N^{T} \otimes M\right] \operatorname{vec}(X) .
$$

Hence, by using these basic operations, the reformulation of the positivity criterium given in Theorem 3.6 goes as follows. The linear matrix inequality in the variable matrix $D \in R^{n \times n}$

$$
\bar{A}+D(I-P) \geq 0
$$

can be reexpressed as the following standard linear inequality

$$
\left[\left(P^{T}-I\right) \otimes I\right] x \leq b,
$$

where $x=\operatorname{vec}(D)$ and $b=\operatorname{vec}(\bar{A})$.

Note that there exist many algorithms that efficiently solve standard LP problems such as simplex method or interior point method. In addition, there are many available softwares that can be used for this purpose, e.g. linprog function in Matlab. One can also use Cplex for large scale systems or the free Sedumi software supported by Yalmip.

\section{Stability Analysis}

In order to provide our main stability result, we shall first state some stability conditions for standard discrete positive systems. We next establish an intermediate stability criterion from which we shall deduce the remarkable fact that for a given positive system (1), its stability for the cone $\operatorname{im}(P) \cap \mathbb{R}_{+}^{n}$ is equivalent to the fact that $\bar{A}$ is a Schur matrix. This result is quite different from the stability result for a continuous positive singular system $[1,29,36]$ for which the associated matrix $\bar{A}$ is not necessarily a Hurwitz matrix.

In the sequel, we make use of the following definition.

Definition 4.1 We say that system (1) is stable for a set $\mathcal{S}$ if for any initial condition $x(0) \in \mathcal{S}, x(k) \rightarrow 0$ as $k \rightarrow \infty$.

The following result will play a key role in the derivation of our main stability result. It includes different equivalent stability conditions.

Proposition 4.2 Let $N$ be a nonnegative matrix and consider the following standard linear system

$$
z(k+1)=N z(k) .
$$

Then the following statements are equivalent.

(a) $N$ is Schur, or equivalently, the system (10) is stable for any initial condition.

(b) There exists $z(0)=z_{0}>0$ such that $z(k)$ goes to zero as $k$ goes to infinity or equivalently

$$
\lim _{k \rightarrow \infty} N^{k} z_{0}=0
$$

(c) There exists $\nu \in \mathbb{R}^{n}$ such that

$$
\nu>0 \text { and }(N-I) \nu<0
$$

(d) There exists $\gamma \in \mathbb{R}^{n}$ such that

$$
\gamma>0 \text { and } \gamma^{T}(N-I)<0 .
$$


(e) $(N-I)^{-1}$ exists and $(N-I)^{-1} \leq 0$.

(f) There exists a definite positive diagonal matrix $Q$ such that the matrix

$$
\bar{A}^{T} Q \bar{A}-Q<0
$$

is definite negative.

Proof. See $[2,15,17]$.

The very special case $P \geq 0$ is the only case that has been extensively investigated in the literature and where positivity and stability conditions for system (1) can be derived in a simple manner, see for instance [5]. Below, we investigate the general case $\operatorname{im}(P) \cap \dot{\mathbb{R}}_{+}^{n} \neq \emptyset$ for which a more elaborated analysis is needed.

Note that the condition $\operatorname{im}(P) \cap \dot{\mathbb{R}}_{+}^{n} \neq \emptyset$ simply means that we require that system $(1)$ admits trajectories that are not just on the boundary of $\mathbb{R}_{+}^{n}$ and therefore it can be considered as a reasonable assumption.

Next, we present a characterization of the stability of system (1) for which the projector $P$ is not necessary nonnegative.

Theorem 4.3 Assume that there exists $v \in \mathbb{R}^{n}$ such that $P v>0$. Then, the following statements are equivalent.

(1) System (1) (or (6)) is positive and stable for the set of admissible initial conditions:

$$
\mathcal{S}=i m(P) \cap \mathbb{R}_{+}^{n} .
$$

(2) There exists a matrix D such that

$$
H:=\bar{A}+D(I-P) \text { is nonnegative and Schur. }
$$

Proof. $(1 \Rightarrow 2)$ : By assumption there exists $x_{0} \in \dot{\mathbb{R}}_{+}^{n} \cap$ $\operatorname{im}(P)$. Since the system is positive we have that $z:=$ $\sum_{k=0}^{T-1} x(k)>0$ with $x(0)=x_{0}$. Using the assumption that $x(k) \rightarrow 0$ as $k \rightarrow \infty$, there exists $T \in \mathbb{N}$ large enough such that $x(T)-x(0)<0$. Hence,

$$
x(T)-x(0)=(\bar{A}-I) \sum_{k=0}^{T-1} x(k)=(\bar{A}-I) z<0 .
$$

Further, note that $P z=z$ because of statement (iii) of Lemma 2.4. Now since the system is positive, by Theorem 3.3, there exists a nonnegative matrix $H$ of the form $H=\bar{A}+D(I-P)$. Moreover, as $(I-P) z=0$ we get

$$
(H-I) z=(\bar{A}-I) z<0,
$$

which implies, by Proposition 4.2, that $H$ is Schur.

$(2 \Rightarrow 1)$ : By statement (ii) in Lemma 2.4, $P x(k)=x(k)$, and therefore we obtain that $x(k+1)=\bar{A} x(k)=H x(k)$ which implies that system $(6)$ is positive and stable for $\mathcal{S}$, as $H$ is nonnegative and Schur.

Remark 4.4 It is worth mentioning that from the proof of Theorem 4.3 it follows that if system (1) is positive and stable for $\mathcal{S}=i m(P) \cap \mathbb{R}_{+}^{n}$ then any matrix of the form $\bar{A}+D(I-P)$ satisfying (8) is necessarily a Schur matrix.

In the following result, we show how we can simultaneously check stability and positivity of system (1) based on LP.

Theorem 4.5 Assume that there exists $v \in \mathbb{R}^{n}$ such that $P v>0$. Then, the following statements are equivalent.

(i) System (1) (or (6)) is positive and stable for the set of admissible initial conditions $\mathcal{S}=i m P \cap \mathbb{R}_{+}^{n}$.

(ii) There exists $\gamma \in \mathbb{R}^{n}, Z \in \mathbb{R}^{n \times n}$ such that

$$
\left\{\begin{aligned}
\gamma^{T}(\bar{A}-I)+\overline{1}_{n}^{T} Z(I-P) & <0 \\
\operatorname{diag}(\gamma) \bar{A}+Z(I-P) & \geq 0 \\
\gamma & >0
\end{aligned}\right.
$$

where $\overline{1}_{n}:=\left[\begin{array}{llll}1 & 1 & \cdots\end{array}\right]^{T} \in \mathbb{R}^{n}$ denotes the unit vector.

Proof. In light of Theorem 4.3 it is enough to show the equivalence between the statement $(i i)$ and the existence of a matrix $D$ such that $\bar{A}+D(I-P)$ is nonnegative and Schur.

By pre-multiplication of the last equation in (12) by $\operatorname{diag}(\gamma)^{-1}$ from the left we obtain that

$$
\bar{A}+\operatorname{diag}(\gamma)^{-1} Z(I-P) \geq 0 .
$$

which is to say that $\bar{A}+D(I-P)$ is nonnegative with $D=$ $\operatorname{diag}(\gamma)^{-1} Z$. Finally, using the fact that $\gamma^{T}=\overline{1} \operatorname{diag}(\gamma)$ and $Z=\operatorname{diag}(\gamma) D$ we have that

$$
\begin{aligned}
\gamma^{T}(\bar{A}-I)+\overline{1}^{T} Z(I-P) & =\gamma^{T}(\bar{A}-I)+\gamma^{T} D(I-P) \\
& =\gamma^{T}(\bar{A}-I+D(I-P))<0 .
\end{aligned}
$$

This, together with $\gamma>0$, is equivalent, by statement (d) of Proposition 4.2, to $\bar{A}+D(I-P)$ being a Schur matrix. The reverse implication can be proved following similar arguments.

Remark 4.6 As we have previously seen, we can solve the matrix inequalities (12) as a standard LP problem. 
We can use the identity $\boldsymbol{v e c}(M X N)=\left[N^{T} \otimes M\right] \boldsymbol{v e c}(X)$ and the fact that for any vector $v$ it holds $\operatorname{diag}(v)=$ $\sum_{i=1}^{n} e_{i} v^{T} e_{i} e_{i}^{T}$, where $e_{i}$ is the $i$-th element of the canonical basis of $\mathbb{R}^{n}$. Hence, it can be easily seen that (12) can be reexpressed as

$$
\begin{aligned}
& {\left[\begin{array}{cc}
\bar{A}^{T} \otimes I & \left(I-P^{T}\right) \otimes \overline{1}_{n} \\
-I & 0
\end{array}\right] x<0} \\
& {\left[-\sum_{i=1}^{n}\left(\bar{A}^{T} e_{i} e_{i}^{T}\right) \otimes e_{i}\left(P^{T}-I\right) \otimes I\right] x \leq 0,} \\
& \text { where the new variable is } x=\left[\begin{array}{c}
\gamma \\
\operatorname{vec}(Z)
\end{array}\right] \text {. }
\end{aligned}
$$

The above linear inequalities involve a strict inequality and non strict one. Since they are homogenous in the vector variable $x$, then a feasible solution can be found by solving the following standard LP problem.

$$
\left[\begin{array}{cc}
\bar{A}^{T} \otimes I & \left(I-P^{T}\right) \otimes \overline{1}_{n} \\
-I & 0 \\
-\sum_{i=1}^{n}\left(\bar{A}^{T} e_{i} e_{i}^{T}\right) \otimes e_{i} & \left(P^{T}-I\right) \otimes I
\end{array}\right] x \leq\left[\begin{array}{c}
-\overline{1}_{n(n+1)} \\
0
\end{array}\right]
$$

Next, we are going to present our main stability result. In fact, based on the previous result of Theorem 4.3, it turns out that the stability condition for the case $P \geq 0$, is also valid for the case $\operatorname{im}(P) \cap \mathbb{R}_{+}^{n} \neq \emptyset$, that is, $\bar{A}$ is a Schur matrix. We show this fact in the following result.

Theorem 4.7 Assume that system (1) is positive and there exists $v \in \mathbb{R}^{n}$ such that Pv $>0$. Then, the following statements are equivalent.

(i) System (1) is positive and stable for the set of admissible initial conditions $\mathcal{S}=i m(P) \cap \mathbb{R}_{+}^{n}$.

(ii) $\bar{A}$ is a Schur matrix.

Proof. Since the implication $(i i) \Rightarrow(i)$ is obvious, we only show the reverse implication. Clearly, it is enough to show that does not exist an unstable eigenvector of $\bar{A}$ that is not in the kernel of $P$. Now, assume that there exists $w \in \mathbb{R}^{n}$ with $P w \neq 0$ such that $\bar{A} w=\alpha w$ with $|\alpha| \geq 1$. Then, by using the identity $\bar{A} P=P \bar{A}$ given by statement (ii) in Lemma 2.4 we obtain $\bar{A} P w=P \bar{A} w=$ $\alpha P w$, which means that $P w$ is also an eigenvector of $\bar{A}$. Next, note that by Theorem 4.3 there exists a matrix $D$ such that $H=\bar{A}+D(I-P)$ is nonnegative and Schur. Thus $H P w=(\bar{A}+D(I-P)) P w$ and by keeping in mind the fact that $P^{2}=P$ (statement (i) of Lemma 2.4) we obtain the following identities

$$
\bar{A} P w=(H P) P w=H P^{2} w=H P w .
$$

Therefore, since we have already seen that $\bar{A} P w=$ $P \bar{A} w=\alpha P w$, it comes $H P w=\alpha P w$, i.e., $P w$ is an unstable eigenvalue of $H$, which is a contradiction to the fact that the matrix $H$ is Schur. Henceforth, necessarily we have that $\bar{A}$ has no unstable eigenvectors and the proof is complete.

In what follows, we show how the previous results can be applied to the very important class of discrete positive singular systems that are described by a Leontief model [25].

A Leontief model of multi-sectors economy is described by

$$
x(k)=L x(k)+C[x(k+1)-x(k)]+d(k),
$$

where $x(k)$ is the vector of output levels, $d(k)$ is the vector of final demands (excluding investments), $L$ is the Leontief input-output matrix and $C$ in the capital coefficient matrix. This model fits into our framework by considering system (1) with $E=C$ and $A=C-L+I$. Thus, based on our previous results, the autonomous Leontief model $(16)(d(k)=0)$ has a unique solution if and only if there exists $\lambda$ such that $(\lambda-1) C+L-I$ is invertible. In this case, define $\widehat{E}=((\lambda-1) C+L-I)^{-1} C$ and $\widehat{A}=((\lambda-1) C+L-I)^{-1}(C-L+I)$, then the system is positive if and only if there exists a matrix $H \geq 0$ such that

$$
\bar{A}=H P,
$$

where $\bar{A}=\widehat{E}^{D} \widehat{A}$ and $P=\widehat{E}^{D} \widehat{E}$.

Example 4.8 Consider the Leontief model given in ([20, Example1]) with no inputs (i.e., $d(k)=0)$ where

$$
C=\left(\begin{array}{ccc}
0.3 & 0.4 & 0.45 \\
0 & 0 & 0 \\
0.6 & 0.8 & 0.9
\end{array}\right), L=\left(\begin{array}{lll}
0.3 & 0.3 & 0.3 \\
0.4 & 0.1 & 0.5 \\
0.3 & 0.5 & 0.2
\end{array}\right)
$$

Since $A=C-L+I$ is invertible, we can select $\lambda=0$. Then, by using Jordan decomposition and formula (3) we can compute the Drazin inverse of $\widehat{E}=-(C+L-I)^{-1} C$ and obtain

$$
\widehat{E}^{D}=\widehat{C}^{D}=-\left(\begin{array}{lll}
0.2347 & 0.3129 & 0.3520 \\
0.2526 & 0.3368 & 0.3789 \\
0.2669 & 0.3559 & 0.4004
\end{array}\right)
$$


By using $\widehat{A}=-I$, the given system can be reexpressed as in the form (6) where its dynamic matrix is given by

$$
\bar{A}=-\widehat{C}^{D}=\left(\begin{array}{lll}
0.2485 & 0.3313 & 0.3727 \\
0.2674 & 0.3566 & 0.4011 \\
0.2826 & 0.3768 & 0.4239
\end{array}\right)
$$

and its associated projector $P$ is given by

$$
P=\widehat{E}^{D} \widehat{E}=\left(\begin{array}{lll}
0.2415 & 0.3220 & 0.3622 \\
0.2599 & 0.3466 & 0.3899 \\
0.2747 & 0.3662 & 0.4120
\end{array}\right) \text {. }
$$

As $P$ and $\bar{A}$ is nonnegative, then this Leontief model is positive. Since $\bar{A}$ is not a Schur matrix $(\sigma(\bar{A}=$ $\{1.0289,0.0000,0.0000\})$, then by using Theorem 4.7, we can conclude that this Leontief model is not stable. This illustrates the fact that a Leontief model is inherently positive and unstable.

\section{$5 \quad$ Internal positivity}

In this section we extend the previous positivity results for autonomous singular systems to singular systems with inputs and outputs described by

$$
\begin{aligned}
E x(k+1) & =A x(k)+B u(k) \\
y(k) & =C x(k)
\end{aligned}
$$

where $E, A \in \mathbb{R}^{n \times n}, B \in \mathbb{R}^{n \times q}$ and $C \in \mathbb{R}^{p \times n}$ are timeinvariant matrices.

In the sequel, we assume that $(E, A)$ is regular and as previously, we use similar notation: $\bar{A}=\widehat{E}^{D} \widehat{A}, \widehat{E}=$ $(\lambda E-A)^{-1} E, \widehat{A}=(\lambda E-A)^{-1} A, \widehat{B}=(\lambda E-A)^{-1} B$ and $P=\widehat{E}^{D} \widehat{E}$.

For ease of notation we also define the following quantities

$$
D_{i}:=-(I-P)\left(\widehat{E} \widehat{A}^{D}\right)^{i} \widehat{A}^{D} \widehat{B} \text { for } i=0, \ldots, \nu-1,
$$

where $\nu$ is the smallest nonnegative integer such that $\operatorname{rank}\left(E^{\nu}\right)=\operatorname{rank}\left(E^{\nu+1}\right)$.

Next, an explicit solution to (17) is stated, see for instance, [7] or [6].

Theorem 5.1 Every solution $x(k)$ of system (17) satisfies

$x(k)=\bar{A}^{k} P v+\sum_{\tau=0}^{k-1} \bar{A}^{k-1-\tau} \widehat{E}^{D} \widehat{B} u(\tau)+\sum_{i=0}^{\nu-1} D_{i} u(k+i)$ for some $v \in \mathbb{R}^{n}$. Moreover, system (17) has a unique solution and it is of the form (19), if and only if the pair $(E, A)$ is regular and its initial conditions $x(0)$ are of the form

$$
x(0)=P v+\sum_{i=0}^{\nu-1} D_{i} u(i)
$$

where $v \in \mathbb{R}^{n}$.

If $(x(0), u(\cdot))$ satisfies $(20)$ we say that $(x(0), u(\cdot))$ is an admissible pair.

Now, let us define precisely the notion of internal positivity.

Definition 5.2 System (17) is said to be internally positive if for any $x(0) \geq 0$ and $u(k) \geq 0$ satisfying equation (20) it follows that $x(k) \geq 0$ and $y(k) \geq 0$ for all $k$.

We are now in a position to state the main result of this section.

Theorem 5.3 System (17) is internally positive if and only if there exists nonnegative matrices $H \geq 0, \widetilde{H} \geq 0$ such that the following conditions hold

$$
\left\{\begin{array}{l}
\bar{A}=H P \\
C \bar{A}=\widetilde{H} P \\
\widehat{E}^{D} \widehat{B}-H D_{0} \geq 0 \\
C \widehat{E}^{D} \widehat{B}-\widetilde{H} D_{0} \geq 0 \\
D_{\nu-1} \geq 0 \\
C D_{\nu-1} \geq 0 \\
D_{i}-H D_{i+1} \geq 0, C D_{i}-\widetilde{H} D_{i+1} \geq 0, i=0, \ldots, \nu-2
\end{array}\right.
$$

where the $D_{i}$ 's matrices have the form (18).

Proof. Necessity: Assume that the system is internally positive. Thus, $x(0)=P v+\sum_{i=0}^{\nu-1} D_{i} u(i) \geq 0$ and $u(0), u(1), \ldots, u(\nu) \geq 0$, i.e.,

$$
\left[\begin{array}{c|cccc}
P & D_{0} & \ldots & D_{\nu-1} & 0 \\
0 & & I
\end{array}\right]\left[\begin{array}{c}
v \\
u(0) \\
\vdots \\
u(\nu)
\end{array}\right] \geq 0
$$

imply that $x(1)=\bar{A} P v+\widehat{E}^{D} \widehat{B} u(0)+\sum_{i=0}^{\nu-1} D_{i} u(i+1) \geq 0$ 
and $y(1)=C x(1) \geq 0$, that is to say

$$
\left[\begin{array}{c|cccc}
\bar{A} P & \widehat{E}^{D} \widehat{B} & D_{0} & \ldots & D_{\nu-1} \\
C \bar{A} P & C \widehat{E}^{D} \widehat{B} & C D_{0} & \ldots & C D_{\nu-1}
\end{array}\right]\left[\begin{array}{c}
v \\
u(0) \\
\vdots \\
u(\nu)
\end{array}\right] \geq 0 .
$$

The result of Lemma 3.2 ensures the existence of a ma$\operatorname{trix} \mathcal{H}=\left[\begin{array}{cccc}H & H_{0} & \ldots & H_{\nu} \\ \widetilde{H} & \widetilde{H}_{0} & \ldots & \widetilde{H}_{\nu}\end{array}\right] \geq 0$ such that

$$
\begin{aligned}
& {\left[\begin{array}{c|cccc}
\bar{A} P & \widehat{E}^{D} \widehat{B} & D_{0} & \ldots & D_{\nu-1} \\
C \bar{A} P & C \widehat{E}^{D} \widehat{B} & C D_{0} & \ldots & C D_{\nu-1}
\end{array}\right]=} \\
& =\mathcal{H}\left[\begin{array}{c|cccc}
P & D_{0} & \ldots & D_{\nu-1} & 0 \\
0 & \multicolumn{2}{|c|}{I}
\end{array}\right] \text {, }
\end{aligned}
$$

or equivalently,

$$
\left\{\begin{array}{l}
\bar{A} P=H P \\
C \bar{A} P=\widetilde{H} P \\
\widehat{E}^{D} \widehat{B}=H D_{0}+H_{0} \\
C \widehat{E}^{D} \widehat{B}=\widetilde{H} D_{0}+\widetilde{H}_{0} \\
D_{\nu-1}=H_{\nu} \\
C D_{\nu-1}=\widetilde{H}_{\nu} \\
D_{i}=H D_{i+1}+H_{i+1} \\
C D_{i}=\widetilde{H} D_{i+1}+\widetilde{H}_{i+1} \text { for } i=0, \ldots, \nu-2 .
\end{array}\right.
$$

Since $\bar{A} P=\bar{A}$ (by statement (ii) of Lemma 2.4) and all the matrices $H_{i}, \widetilde{H}_{i}$ are nonnegative, then the conditions (21) of the theorem readily follow.

Sufficiency: Let $x(0)=P v+\sum_{i=0}^{\nu-1} D_{i} u(i) \geq 0$ and $u(k) \geq$ 0 be given such that $(x(0), u(k))$ is an admissible pair. We shall prove that $x(k)$ is nonnegative for all $k$ by induction. First, let us show that $x(1)$ is nonnegative. Since $H \geq 0$ and $D_{\nu-1} \geq 0$.

$$
\begin{aligned}
& x(1)=\bar{A} P v+\widehat{E}^{D} \widehat{B} u(0)+\sum_{i=0}^{\nu-1} D_{i} u(i+1) \\
& x(1) \geq H P+H D_{0} u(0)+\sum_{i=0}^{\nu-2} H D_{i+1} u(i+1)+D_{\nu-1} u(\nu) \\
& x(1) \geq H P+\sum_{i=0}^{\nu-1} H D_{i} u(i)+D_{\nu-1} u(\nu)
\end{aligned}
$$

Hence, it follows that

$$
x(1) \geq H\left[P+\sum_{i=0}^{\nu-1} D_{i} u(i)\right]=H x(0) \geq 0 .
$$

Assume now that $x(k)=\bar{A}^{k} P v+\sum_{\tau=0}^{k-1} \bar{A}^{k-\tau-1} \widehat{E}^{D} \widehat{B} u(\tau)+$ $\sum_{i=0}^{\nu-1} D_{i} u(k+i)$ is nonnegative. At this stage it suffices to show that $x(k+1) \geq H x(k)$ and as $H \geq 0$ then we can conclude that $x(k+1) \geq 0$. This fact goes as follows.

$$
\begin{aligned}
& x(k+1) \\
= & \bar{A}^{k+1} \widehat{E}^{D} \widehat{E} v+\sum_{\tau=0}^{k} \bar{A}^{k-\tau} \widehat{E}^{D} \widehat{B} u(\tau)+\sum_{i=0}^{\nu-1} D_{i} u(k+1+i) \\
= & \overline{A A}^{k} \widehat{E}^{D} \widehat{E} v+\sum_{\tau=0}^{k-1} \overline{A A}^{k-\tau-1} \widehat{E}^{D} \widehat{B} u(\tau)+ \\
& +\widehat{E}^{D} \widehat{B} u(k)+\sum_{i=0}^{\nu-1} D_{i} u(k+1+i) \\
\geq & \bar{A}\left[\bar{A}^{k} \widehat{E}^{D} \widehat{E} v+\sum_{\tau=0}^{k-1} \bar{A}^{k-\tau-1} \widehat{E}^{D} \widehat{B} u(\tau)\right]+H D_{0} u(k)+ \\
& +\sum_{i=0}^{\nu-2} H D_{i+1} u(k+1+i)+D_{\nu-1} u(k+\nu) \\
= & \bar{A}\left[\bar{A}^{k} \widehat{E}^{D} \widehat{E} v+\sum_{\tau=0}^{k-1} \bar{A}^{k-\tau-1} \widehat{E}^{D} \widehat{B} u(\tau)\right]+ \\
& +\sum_{i=0}^{\nu-1} H D_{i} u(k+i)+D_{\nu-1} u(k+\nu) \\
\geq & \bar{A}\left[\bar{A}^{k} \widehat{E}^{D} \widehat{E} v+\sum_{\tau=0}^{k-1} \bar{A}^{k-\tau-1} \widehat{E}^{D} \widehat{B} u(\tau)\right]+ \\
& +\sum_{i=0}^{\nu-1} H D_{i} u(k+i) .
\end{aligned}
$$

Taking into account that $\bar{A}=H P$ we have

$$
\begin{aligned}
x(k+1) \geq & H P\left[\bar{A}^{k} \widehat{E}^{D} \widehat{E} v+\sum_{\tau=0}^{k-1} \bar{A}^{k-\tau-1} \widehat{E}^{D} \widehat{B} u(\tau)\right] \\
= & +\sum_{i=0}^{\nu-1} H D_{i} u(k+i) .
\end{aligned}
$$

Next, note that $\widehat{E}^{D} \widehat{E}^{D} \widehat{E}=\widehat{E}^{D}$, i.e., $P \widehat{E}^{D}=\widehat{E}^{D}$. From this, together with the fact that $P \bar{A}=\bar{A} P=P$ (see 
statement (ii) of Lemma 2.4) it holds that

$$
\begin{aligned}
& x(k+1) \\
\geq & H P\left[\bar{A}^{k} \widehat{E}^{D} \widehat{E} v+\sum_{\tau=0}^{k-1} \bar{A}^{k-\tau-1} \widehat{E}^{D} \widehat{B} u(\tau)+\sum_{i=0}^{\nu-1} D_{i} u(k+i)\right] \\
= & H\left[\bar{A}^{k} \widehat{E}^{D} \widehat{E} v+\sum_{\tau=0}^{k-1} \bar{A}^{k-\tau-1} \widehat{E}^{D} \widehat{B} u(\tau)+\sum_{i=0}^{\nu-1} D_{i} u(k+i)\right] \\
= & H x(k) \geq 0 .
\end{aligned}
$$

The proof of $y(k)=C x(k) \geq 0$ follows the same lines of arguments, and therefore it is skipped. This concludes the proof.

Remark 5.4 We can reformulate conditions (21) in the variables $H$ and $\widetilde{H}$ as a unique standard LP problem by using the vec and Kronecker operations as it was done previously for the LP problem (9).

Hereafter, we show how our general result given by conditions (21) can be simplified for the case $P \geq 0$. Specifically, for this case, our result can be viewed as a completion of Theorem 3.8 in [36] and Proposition 1 in [5]. We stress out that in [5] only the case $C=I$ was treated. Also in [36] the characterization of internal positivity uses a condition on the matrix $C$ that cannot be directly checked.

In the case $P \geq 0$, we provide an explicit and simplified characterization for internal positivity of system (17) that solely involves its dynamic matrices.

Corollary 5.5 Assume $P \geq 0$. Then, system (17) is internally positive if and only if the following conditions hold

$$
\left\{\begin{array}{l}
\bar{A} \geq 0 \\
C \bar{A} \geq 0 \\
\widehat{E}^{D} \widehat{B} \geq 0 \\
C \widehat{E}^{D} \widehat{B} \geq 0 \\
D_{i} \geq 0, C D_{i} \geq 0 \text { for } i=0, \ldots, \nu-1 .
\end{array}\right.
$$

Proof. Necessity: Clearly, by using the conditions (21) in Theorem 5.3, as $P \geq 0$ and $H \geq 0, \widetilde{H} \geq 0$ this implies that $\bar{A} \geq 0$ and $C \bar{A} \geq 0$. Also, since $D_{\nu-1} \geq 0$ we have that $D_{i} \geq 0$ for $i=0, \ldots, \nu-1$ and thus it holds also $\widehat{E}^{D} \widehat{B} \geq 0, C \widehat{E}^{D} \widehat{B} \geq 0$.

Sufficiency: Since $P v=P x(0), x(k)$ can be expressed as

$x(k)=\bar{A}^{k} P x(0)+\sum_{\tau=0}^{k-1} \bar{A}^{k-1-\tau} \widehat{E}^{D} \widehat{B} u(\tau)+\sum_{i=0}^{\nu-1} D_{i} u(k+i)$.
As $P \geq 0, \bar{A} \geq 0, \widehat{E}^{D} \widehat{B} \geq 0$ and $D_{i} \geq 0$ for $i=0, \ldots, \nu$ then, obviously $x(k) \geq 0$. In the same manner we notice that

$$
\begin{aligned}
y(k) & =(C \bar{A}) \bar{A}^{k-1} P x(0)+\sum_{\tau=0}^{k-2}(C \bar{A}) \bar{A}^{k-2-\tau} \widehat{E}^{D} \widehat{B} u(\tau)+ \\
& +C \widehat{E}^{D} \widehat{B} u(k-1)+\sum_{i=0}^{\nu-1} C D_{i} u(k+i),
\end{aligned}
$$

since $C \bar{A} \geq 0, \bar{A} \geq 0, C \widehat{E}^{D} \widehat{B} \geq 0$ and $C D_{i} \geq 0$ for $i=0, \ldots, \nu$ then, it is straightforward to see that $y(k)$ is nonnegative.

\section{Conclusions}

We have provided an efficient approach to the analysis of discrete positive singular systems. The derived conditions for which a singular system is positive or internally positive are necessary and sufficient. The stability analysis of autonomous positive singular systems was also addressed, and different stability criteria were provided. In addition, we have shown that all the proposed conditions can be reformulated and efficiently solved in terms of linear programming. Our future work concerns the stabilization issue. Due to the positivity constraint the stabilization problem can be very difficult to solve within the proposed framework.

\section{Acknowledgement}

The authors are very grateful to the anonymous reviewers and the Associate Editor for their comments and suggestions which led to significant improvements.

\section{References}

[1] M. Ait Rami and D. Napp. Characterization and stability of autonomous positive descriptor systems. IEEE Transactions on Automatic Control, 57(10):2668-2673, 2012.

[2] M. Ait Rami and F. Tadeo and A. Benzaouia. Control of constrained positive discrete systems. American Control Conference ACC,:5851-5856, 2007

[3] G. I. Bara. Robust analysis and control of parameterdependent uncertain descriptor systems. Systems $\&$ Control Letters, 60(5):356-364, 2011.

[4] R. Bru, C. Coll, S. Romero-Vivo, and E. Sánchez. Some problems about structural properties of positive descriptor systems. In Positive systems (Rome, 2003), volume 294 of Lecture Notes in Control and Inform. Sci., pages 233-240. Springer, Berlin, 2003.

[5] R. Bru, C. Coll, and E. Sánchez. Structural properties of positive linear time-invariant difference-algebraic equations. Linear Algebra Appl., 349:1-10, 2002.

[6] T. Brüll. Explicit solutions of regular linear discrete-time descriptor systems with constant coefficients. Electron. J. Linear Algebra, 18:317-338, 2009. 
[7] S. L. Campbell. Singular systems of differential equations, volume 40 of Research Notes in Mathematics. Pitman (Advanced Publishing Program), Boston, Mass., 1980.

[8] S. L. Campbell and C. D. Meyer, Jr. Generalized inverses of linear transformations. Dover Publications Inc., New York, 1991. Corrected reprint of the 1979 original.

[9] B. Cantó, C. Coll, and E. Sánchez. Drazin inverse and periodic collection of matrices. SIAM. J. Matrix Anal. and Appl., 27(2):413-423, 2005.

[10] B. Cantó, C. Coll, and E. Sánchez. Positive solutions of a discrete-time descriptor system. Internat. J. Systems Sci., 39(1):81-88, 2008.

[11] V. Chellaboina, W. M. Haddad, and Q. Hui. Nonnegative and Compartmental Dynamical Systems. NJ: Princeton University Press, 2010.

[12] L. Dai. Singular control systems, volume 118 of Lecture Notes in Control and Information Sciences. Springer-Verlag, Berlin, 1989.

[13] M. P. Drazin. Pseudo-inverses in associative rings and semigroups. Amer. Math. Monthly, 65:506-514, 1958.

[14] G. R. Duan. Analysis and Design of Descriptor Linear Systems. Springer, 2010.

[15] L. Farina and S. Rinaldi. Positive linear systems. Pure and Applied Mathematics (New York). Wiley-Interscience, New York, 2000. Theory and applications.

[16] F. Ferranti, D. De Zutter, L. Knockaert, T. Dhaene. Model order reduction with preservation of passivity, nonexpansivity and markov moments. Systems \& Control Letters, 60(1):53-61, 2011.

[17] E. Fornasini, M.E. Valcher. Stability and stabilizability criteria for discrete-time positive switched systems. IEEE Transactions on Automatic Control, 57(5):1208-1221, 2012.

[18] A. Herrero, A. Ramírez, and N. Thome. An algorithm to check the nonnegativity of singular systems. Appl. Math. Comput., 189(1):355-365, 2007.

[19] A. Herrero, A. Ramírez, and N. Thome. Nonnegativity, stability, and regularization of discrete-time descriptor systems. Linear Algebra Appl., 432(4):837-846, 2010.

[20] L. Jódar and P. Merello. Positive solutions of discrete dynamic Leontief input-output model with possibly singular capital matrix. Math. Comput. Modelling, 52(7-8):1081-1087, 2010.

[21] T. Kaczorek. Positive $1 D$ and $2 D$ systems. Springer-Verlag, London, 2005.

[22] P. Kunkel and V. Mehrmann. Differential-algebraic equations. Analysis and numerical solution. EMS Textbooks in Mathematics. European Mathematical Society (EMS), Zürich, 2006.

[23] J. Lam, S. Xu. Robust Control and Filtering of Singular Systems. Springer, 2006.

[24] F. L. Lewis. A survey of linear singular systems. Circuits Systems Signal Process., 5(1):3-36, 1986.

[25] W. W. Leontief. Input-Output Economics. Oxford University Press, 1966.

[26] D. G. Luenberger. Introduction to Dynamic Systems. John Wiley, 1979.

[27] O. L. Mangasarian. Characherizations of real matrices of monotone kind. SIAM Review, 10(4):439-441, 1968.

[28] J. W. Nieuwenhuis. About Positive Invariance and Asymptotic Stability. Appl Math Optim, 12:81-87, 1984.
[29] T. Reis and E. Virnik. Positivity preserving balanced truncation for descriptor systems. SIAM J. Control Optim., 48(4):2600-2619, 2009.

[30] R. Riaza. Differential-algebraic systems. Analytical aspects and circuit applications. World Scientific Publishing Co. Pte. Ltd., Hackensack, NJ, 2008.

[31] P. Shi, Z. Yan. Disturbance impulse controllability in descriptor system. IEEE Transactions on Automatic Control, 56(4):946-951, 2011.

[32] M. S. Silva and T. P. de Lima. Looking for nonnegative solutions of a Leontief dynamic model. Linear Algebra Appl., 364:281-316, 2003.

[33] J. R. Stern. A note on positively invariant cones. Appl Math Optim, 9:67-72, 1982.

[34] A. Steinbrecher, T. Stykel, M. Hinze, M. Kunkel. Model order reduction of coupled circuit-device systems. International Journal of Numerical Modelling: Electronic Networks, Devices and Fields, 25(4):362-377, 2012.

[35] E. Virnik. Analysis of Positive Descriptor Systems: Topics in Systems and Control Theory. Springer, 2008.

[36] E. Virnik. Stability analysis of positive descriptor systems. Linear Algebra Appl., 429(10):2640-2659, 2008.

[37] W. Yimin. A characterization and representation of the drazin inverse. SIAM. J. Matrix Anal. and Appl., 17(4):744$747,1996$.

[38] L. Zhang. A characterization of the Drazin inverse. Linear Algebra Appl., 335:183-188, 2001. 